\title{
Studies on thermo-optic property of chitosan-alizarin yellow GG complex: a direction for devices for biomedical applications
}

\author{
NIDHI NIGAM, SANTOSH KUMAR ${ }^{\#}$, PRADIP KUMAR DUTTA and TAMAL GHOSH* \\ Department of Chemistry, Motilal Nehru National Institute of Technology, Allahabad 211 004, India \\ \#Present address: Department of Chemistry, Coimbra University, Coimbra, Portugal
}

MS received 18 May 2015; accepted 16 June 2015

\begin{abstract}
The optical parameters including the refractive index $(n)$ and thermo-optic coefficient, TOC (dn/dT), the dielectric constant $(\varepsilon)$ and its variation with temperature, and the thermal volume expansion coefficient $(\beta)$ and its variation with temperature of chitosan-alizarin yellow GG (CS-AY GG) complex were examined. The dn/dT and $\varepsilon$ values for the polymer derivative were in the range $-2.5 \times 10^{-4}$ to $1.2 \times 10^{-4 \circ} \mathrm{C}^{-1}$ and 2.2 to 2.3 , respectively. The $\mathrm{d} n / \mathrm{d} T$ values were larger than that of inorganic glasses such as zinc silicate glass $\left(5.5 \times 10^{-6 \circ} \mathrm{C}^{-1}\right)$ and borosilicate glass $\left(4.1 \times 10^{-6 \circ} \mathrm{C}^{-1}\right)$ and were larger than that of organic polymers such as polystyrene $\left(-1.23 \times 10^{-4 \circ} \mathrm{C}^{-1}\right)$ and PMMA $\left(-1.20 \times 10^{-4 \circ} \mathrm{C}^{-1}\right)$. The $\varepsilon$-values are lower than optically estimated $\varepsilon$-values of conventional polymer (3.00), aliphatic polyimide (2.5) and semi-aromatic polyamide (2.83). The obtained results of chitosan derivative are expected to be useful for optical switching and optical waveguide areas for devices of biomedical applications.
\end{abstract}

Keywords. Thermo-optic property; SHG; chitosan-alizarin complex; biomedical; applications.

\section{Introduction}

Polymer-based azomaterials have drawn great attention in the past decades due to their vast potential applications in high technology fields, such as optical information transmission, optical data storage, optical switching and nonlinear optical (NLO) materials. ${ }^{1-6}$ Along with the successful invention of lasers, rapid development in optical communication has caused the larger requirement for optical control and optical transfer devices. Polymeric NLO materials offer their potential applications for lower switching voltage and higher bandwidth electro-optic devices and have been a subject of intense research interest in recent years. Advantages of the NLO polymers in properties such as high electro-optic constants and low dielectric constant have now been extensively demonstrated. ${ }^{7-10}$ For polymeric-based devices to effectively compete with inorganic materials, issues such as optical insertion loss and thermal stability have to be improved.

In recent times, polymer-based optical waveguides for fabrication of thermo-optic devices have attracted great interest in the telecommunication application areas such as digital optical switch, Mach-Zehnder interferometer-type optical switch and optical cross-connects due to their high thermooptic coefficient (TOC) of the polymer, which is one order of magnitude larger than those for silica material. ${ }^{11-13}$ Therefore, it is important to study the thermo-optic properties of the polymeric material.

\footnotetext{
*Author for correspondence (tamalghosh@mnnit.ac.in)
}

With increasing importance of optical active polymer compounds in the application field of drug, pesticide and food industries, there are strict regulations stemming from the realization that the mirror image of an active compound may have a devastatingly deleterious effect. ${ }^{14-17}$ The optical property when induced in a biopolymer makes it important in terms of its applicability. The amino group of chitosan is also known to form ionic complexes with several anionic dyes. The optical group acts as a most important functionality and thus, responsible for NLO activity due to the presence of azo group. Han reported the induced chirality of a NLO chromophores and the chiral chitosan polymer complex as a potential candidate for the second-order NLO material. ${ }^{18}$

In the last few years, interests have increased considerably in the naturally occurring biopolymers owing to their abundance, nontoxicity and potential substitute for useful biomedical applications. This paper focuses on the biopolymer chitosan and the optical properties of its derivatives. Chitosan, beta-( $1 \rightarrow 4)$-2-amino-2-deoxy-D-glucopyranose, is a weak cationic polysaccharide produced by deacetylation of natural polymer chitin and has many useful biological properties, such as biocompatibility, biodegradability and bioactivity. ${ }^{19-24}$ Because of the existence of amine groups, chitosan is a polycation and is able to form intermolecular complexes with a wide variety of polyanions, including metals, dyes and proteins. In this paper, the optical parameters including the refractive index $(n)$ and TOC $(\mathrm{d} n / \mathrm{d} T)$, the dielectric constant $(\varepsilon)$ and its variation with temperature, and the thermal volume expansion coefficient $(\beta)$ and its variation with temperature of chitosan-alizarin yellow GG (CS-AY GG) complex are examined for its suitability in optical devices in biomedical applications. 


\section{Materials and methods}

The refractive index $(n)$ values of the chitosan polymer solutions with different degrees of deacetylation (DD) were measured with an Abbe' refractometer (WAY-2S, Nanjing Li'er Lab Instrument Equipment Co., Ltd., China) in the temperature interval $13.0-70.0^{\circ} \mathrm{C}$ under normal pressure. The temperature was controlled by circulating water into the refractometer through a thermostatically controlled bath with a digital temperature control unit to maintain the desired temperature within $\pm 0.01{ }^{\circ} \mathrm{C}$. The apparatus was calibrated by measuring Millipore quality water and toluene before measurements. Samples were directly introduced into the cell with a syringe. At least seven independent measurements were taken for each sample at each temperature to assure the effectiveness of the measurement. Refractive index $(n)$ values were measured to an accuracy of \pm 0.0001 after the sample mixture was thermostatically controlled at equilibrium. To obtain consistent values, the temperature was held constant throughout each set of measurements and every experiment was repeated three times under the same condition. The refractive index values of polymer solutions with different DD values at different temperatures were measured (table 1). Different degrees of deacetylation of chitosan (79, 90 and 95\%) were used for obtaining respective CS-AY GG complexes for studying refractive index measurements. These complexes were dissolved in DMSO and prepared to the solution whose mass fraction was $10 \%$ with the aid of ultrasonication instrument in the frequency of $77 \mathrm{~Hz}$ for $20 \mathrm{~min}$. The refractive indices of the derivatives were measured at complex light and different temperatures.

Chitosan powder was mixed into isopropyl alcohol/water mixture of acetic acid and slowly stirred at room temperature, then the mixture was subsequently kept in the given reaction temperature and time followed by washing and drying the desired product, CS-AY GG complex was formed as described in our earlier publication. ${ }^{25}$ The characterization of the derivative has been described elsewhere and it is described here in brief. $^{25}$

\section{Results}

The FTIR spectrum of CS-AY GG complex shows peak at 3464, 3097, 2925, 2715, 1648, 1598, 1527, 1446, 1345, 1139 and $1087 \mathrm{~cm}^{-1}$. The ${ }^{1} \mathrm{H}$ NMR spectrum of CS-AY GG complex shows singlet at 8.47, 8.32, 3.35, 4.02, $2.50 \mathrm{ppm}$ and multiplet in the range $1.19-1.16 \mathrm{ppm}$ in DMSO- $d_{6}$ solvent. The TGA thermogram of CS-AY GG complex shows that the polymer has $2.5 \%$ weight loss at $105^{\circ} \mathrm{C}$ and starts to degrade at $261^{\circ} \mathrm{C}$. The rate of weight loss increases with the increase in temperature and attains a maximum value at $285^{\circ} \mathrm{C} . T_{\max }$, temperature at which maximum degradation has been found, is $285^{\circ} \mathrm{C}$. Second $T_{\max }$ has been found at about $352^{\circ} \mathrm{C}$. The scanning electron micrographs (SEMs) of the azo-based chitosan derivative exhibits a non-porous, non-smooth membranous phases consisting of fibres. It also displays rod-shaped and tube-shaped structure. The UV-vis spectrum of the CSAY GG complex shows a broad band between the 394 and $404 \mathrm{~nm}$ in DMSO. The photoluminescence spectrum of CSAY GG complex shows emission band at 483, 526 and $600 \mathrm{~nm}$ upon excitation at $254 \mathrm{~nm}$. The CS-AY GG complex's second harmonic (SHG) signal of $450 \mathrm{mV}$ was obtained, while the KDP gave an SHG signal of $140 \mathrm{mV}$ for the same input beam energy.

\section{Discussions}

In the FTIR spectrum of CS-AY GG complex, the vibrational band at $3464 \mathrm{~cm}^{-1}$ is due to axial OH group of chitosan and alizarin yellow and bands at 3097, 2925, 1139 and $1087 \mathrm{~cm}^{-1}$ corresponds to symmetric or asymmetric $\mathrm{CH}_{2}$ stretching vibration. The other important vibrational bands are $2715 \mathrm{~cm}^{-1}$ (CN asymmetric band stretching), $1648 \mathrm{~cm}^{-1}$ $\left(\mathrm{COO}^{-}\right.$asymmetric stretching), $1527 \mathrm{~cm}^{-1}$ (symmetrical bending stretching of amine salt $\left.\mathrm{NH}_{3}^{+}\right), 1345 \mathrm{~cm}^{-1}(\mathrm{CN}$ band stretching, axial deformation of amino group) and $1446 \mathrm{~cm}^{-1}$ (stretching vibration of $\mathrm{NO}_{2}$ group), and the $\mathrm{N}=\mathrm{N}$ (azo) linkage is indicated by the peak at $1598 \mathrm{~cm}^{-1}$. Degree of substitution (DS) also affects the intensity of the $\mathrm{OH}$ band. $\mathrm{OH}$ stretching becomes broader and moves to a higher frequency with the increase in DS up to $\sim 80 \%$, indicating an increase in the disordered structure.

The broader small angle peaks in chitosan suggests that chitosan derivative exhibits higher long-range order. Chitosan derivative has higher intensity pattern than chitosan, which indicates that chitosan is substantially more amorphous. XRD pattern proves that the crystal lattice has transformed from amorphous structure into a relatively crystalline structure in CS-AY GG complex. ${ }^{26}$

For ${ }^{1} \mathrm{H}$ NMR (DMSO- $d_{6}$ ) spectra of CS-AY GG complex, $\delta=8.32 \mathrm{ppm}$ (s) is due to $\mathrm{OH}$ proton, $\delta=8.47 \mathrm{ppm}$ (s) due to $\mathrm{NH}_{3}^{+}$proton of the amine salt, $\delta=3.35 \mathrm{ppm}$ (broad, s) due to $\mathrm{CH}_{2}$ group of $\mathrm{CH}_{2} \mathrm{OH}$ of chitosan, $\delta=4.02$ $\mathrm{ppm}(\mathrm{s})$ is due $\mathrm{OH}$ of alizarin and $\delta=1.19-1.16 \mathrm{ppm}(\mathrm{m})$, $2.50 \mathrm{ppm}$ (broad, s) is due to glucosamine residue of chitosan. Compared with chitosan, CS-AY GG complex shows new proton signal at $\delta=8.47 \mathrm{ppm}$, which confirms the complex formation between $\mathrm{NH}_{3}^{+}$group of chitosan and $\mathrm{COO}^{-}$ group of alizarin yellow under mild condition.

The TGA thermogram of CS-AY GG complex shows that the polymer has $2.5 \%$ weight loss at $105^{\circ} \mathrm{C}$ due to the loss of absorbed water and started to degrade at $261^{\circ} \mathrm{C} . T_{\max }$, temperature for maximum degradation, is $285^{\circ} \mathrm{C}$ for degradation of chitosan chain. Second $T_{\max }$ at about $352^{\circ} \mathrm{C}$ is due to the lower grafting of acid in a polymer. The second step of degradation supports that the CS-AY GG complex is thermally more stable than pure chitosan.

The non-porous, non-smooth membranous phases with rod-shaped and tube-shaped structures in the SEM of the azobased chitosan derivative indicates that the maximum fluorescent intensity comes from the tubes. This property may be an added advantage for the biomedical applications of the optical material. 
Table 1. Refractive index data of polymer samples with different DD values.

\begin{tabular}{lccccccc}
\hline \multirow{2}{*}{$\begin{array}{l}\text { Polymer with different } \\
\text { DD values (\%) }\end{array}$} & \multicolumn{6}{c}{ Refractive index at different temperatures $\left({ }^{\circ} \mathrm{C}\right)$} \\
\cline { 2 - 8 } 79 & 13 & 20 & 30 & 40 & 50 & 60 & 70 \\
\hline 90 & 1.5136 & 1.5124 & 1.5109 & 1.5100 & 1.5094 & 1.5084 & 1.5058 \\
95 & 1.5388 & 1.5381 & 1.5370 & 1.5350 & 1.5331 & 1.5308 & 1.5297 \\
& 1.5403 & 1.5397 & 1.5380 & 1.5352 & 1.5311 & 1.5291 & 1.5270 \\
\hline
\end{tabular}

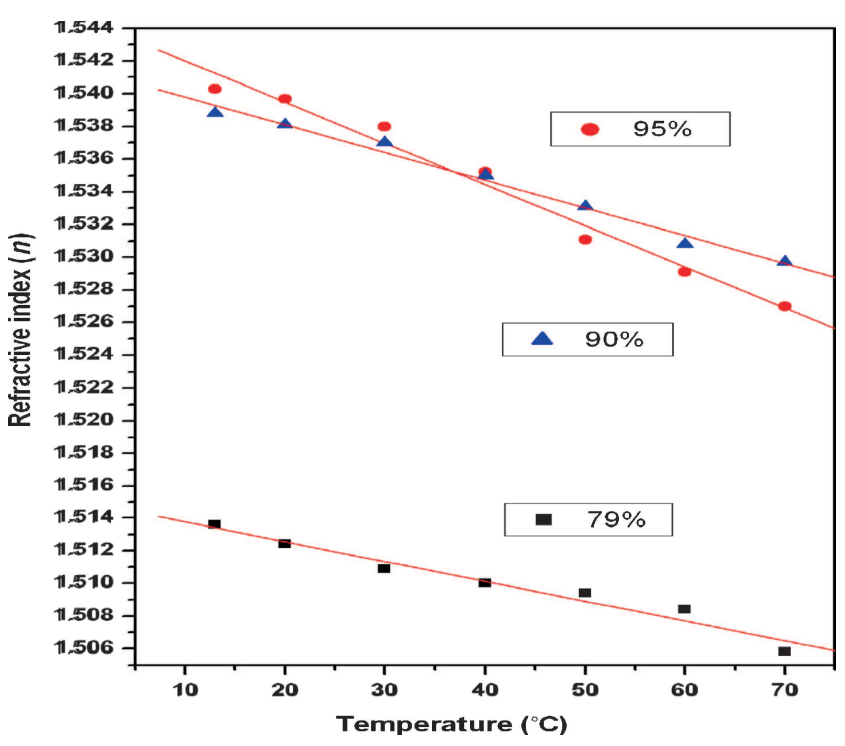

Figure 1. Refractive index variation with temperature of $\mathrm{CS}-\mathrm{AY}$ GG complex.

The broad absorption band in the UV-vis spectrum of the CS-AY GG complex is due to its $\pi-\pi^{*}$ transition. The CS-AY GG complex shows red-shifted emission maxima due to the introduction of side chain and the electronic effect of the substituent on the side chain because the attachment of the conjugate side group to the backbone will enlarge the degree of delocalized $\pi$-bond on the electron-rich polymer main chain. The emission intensity was greatly influenced by the conjugation length and variation of the substituents. The $\mathrm{p}-\pi$ conjugated side chain can make the emission maximum of CS-AY GG complex red shift. The shifting wavenumber is decided by electronic effect. The side chain containing the electron-withdrawing group will decrease the emission intensity while the electron donating group will increase it. ${ }^{27}$

The CS-AY GG complex SHG signal of $450 \mathrm{mV}$ was obtained, while the KDP gave an SHG signal of $140 \mathrm{mV}$ for the same input beam energy. Thus SHG relative efficiency of CS-AY GG complex was found to be 3.2 times higher than that of KDP. ${ }^{28,29}$

The refractive index values of different contents of CSAY GG complex at different temperatures were measured (table 1) and graphical presentation for $\mathrm{d} n / \mathrm{d} T$ are shown in figure 1 . The $\varepsilon$ and the volume expansion coefficient were calculated from the following relation ${ }^{30}$ and are shown in figures 2 and 3 . The dielectric constant $(\varepsilon)$ was calculated according to the following relationship between $n$ and $\varepsilon$ :

$$
\varepsilon=n^{2},
$$

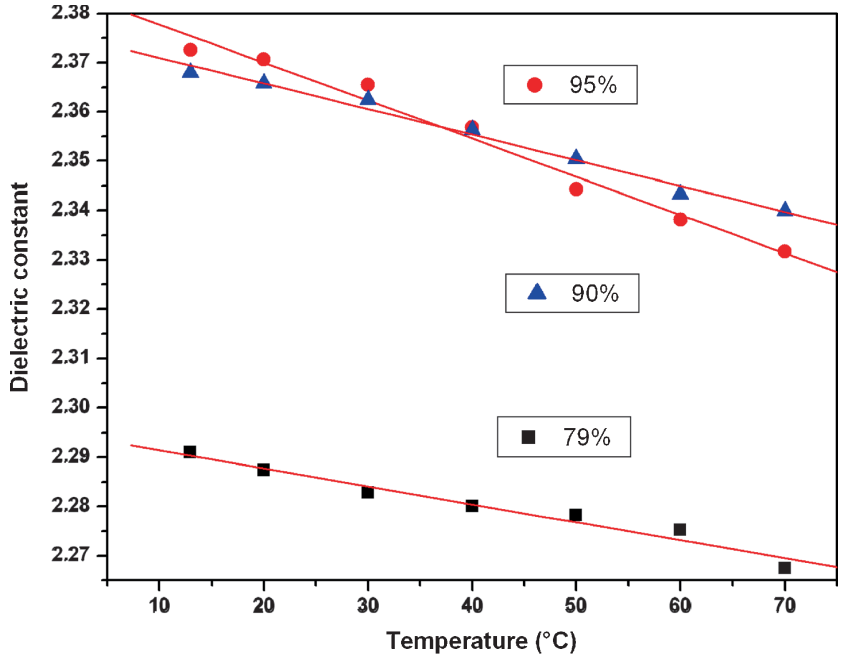

Figure 2. Dielectric constant variation with temperature of CSAY GG complex.

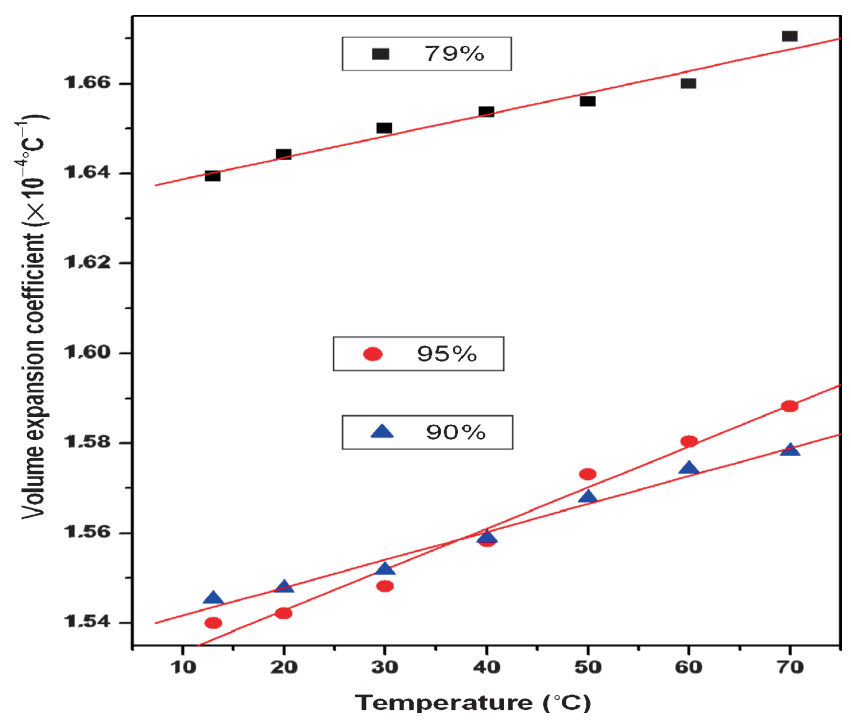

Figure 3. Thermal volume expansion with temperature of CSAY GG complex.

$\beta$ is the thermal volume expansion coefficient, which can be calculated according to $f(n)$ and $\mathrm{d} n / \mathrm{d} T$ by the equation

$$
\mathrm{d} n / \mathrm{d} T=-f(n) \beta
$$

with

$$
f(n)=\frac{\left(n^{2}-1\right)\left(n^{2}+2\right)}{6 n} .
$$


Table 2. Optical properties of CS-AY GG complex.

\begin{tabular}{lccc}
\hline & \multicolumn{3}{c}{ Chitosan polymer content (DD \%) } \\
\cline { 2 - 4 } Property & $79 \%$ & $90 \%$ & $95 \%$ \\
\hline Thermo-optic & -1.21175 & -1.6955 & -2.51979 \\
$\mathrm{~d} n / \mathrm{d} T\left(\times 10^{-4 \circ} \mathrm{C}^{-1}\right)$ & -0.98147 & -0.99372 & -0.99004 \\
Correlation co-efficient & 5.42275 & 4.37685 & 8.21548 \\
$\quad$ Standard deviation $\left(\times 10^{-4}\right)$ & & & \\
Dielectric & -3.65914 & -5.20299 & -7.73041 \\
$\mathrm{~d} \varepsilon / \mathrm{d} T\left(\times 10^{-4 \circ} \mathrm{C}^{-1}\right)$ & -0.98154 & -0.99376 & -0.99008 \\
Correlation co-efficient & 1.63 & 1.34 & 2.52 \\
Standard deviation $\left(\times 10^{-3}\right)$ & & & 6.1385 \\
Thermal expansion & 4.81679 & 9.13838 & 0.98979 \\
$\mathrm{~d} \beta / \mathrm{d} T\left(\times 10^{-8 \circ} \mathrm{C}^{-1}\right)$ & 0.98111 & 0.99345 & 3.01732 \\
Correlation co-efficient & 2.17745 & 1.61907 & \\
Standard deviation $\left(\times 10^{-7}\right)$ & & & \\
\hline
\end{tabular}

The slope of the $n$ curve $(\mathrm{d} n / \mathrm{d} T)$ was found to be $-2.5 \times$ $10^{-4}$ to $1.2 \times 10^{-4 \circ} \mathrm{C}^{-1}$ (figure 1 ). The correlative coefficient was -0.9814 to -0.990 , which indicated that $n$ and temperature had a good linear correlation in the selected temperature range. The standard deviation was very low. The TOCs of the obtained CS-AY GG complex were one order of magnitude larger than those of inorganic glasses such as zinc silicate glass $\left(5.5 \times 10^{-6 \circ} \mathrm{C}^{-1}\right)$ and borosilicate glass $\left(4.1 \times 10^{-6 \circ} \mathrm{C}^{-1}\right)$ and were larger than polystyrene $(-1.23 \times$ $\left.10^{-4 \circ} \mathrm{C}^{-1}\right)$ and PMMA $\left(-1.20 \times 10^{-4 \circ} \mathrm{C}^{-1}\right)$.

As shown in figure 2 and table 2, the variation of the dielectric constant with temperature $(\mathrm{d} \varepsilon / \mathrm{d} T)$ of CS-AY GG complex made from different $\mathrm{DD} \%$ values of polymer was $-7.7 \times 10^{-4}$ to $-3.6 \times 10^{-4 \circ} \mathrm{C}^{-1}$. The correlative coefficient was -0.9900 to -0.9815 which indicated that $\varepsilon$ and temperature had a good linear correlation in the selected temperature range. The standard deviation was very low. In microelectronic device circuits, the propagation velocity of the signal is inversely proportional to the square of $\varepsilon$ of the propagation medium. Therefore, materials with the low $\varepsilon$ are required for faster signal propagation in microelectronic devices. ${ }^{31}$ In this experiment, the obtained $\varepsilon$-values for the polymer are in the range $2.2-2.3$. The values are lower than optically estimated $\varepsilon$-values of conventional polymer (3.00), ${ }^{32}$ aliphatic polyimide (2.5) and semi-aromatic polyamide (2.83). ${ }^{33}$ The results indicate that the obtained polymer derivative could be expected to be applied in optical switches, waveguide fields and the microelectronics chips as a dielectric layer.

The possibility of controlling the coefficient of thermal expansion in pure and composite materials has been a principal driving force. As shown in figure 3 and table 2, the variation of the thermal volume expansion coefficient with temperature $(\mathrm{d} \beta / \mathrm{d} T)$ of derivative was $4.8 \times 10^{-8}$ to $6.1 \times$ $10^{-8 \circ} \mathrm{C}^{-1}$. The correlative coefficient was $0.9811-0.9934$, which indicated that $\beta$ and temperature had a good linear correlation in the selected temperature range. The standard deviation was very low.

Figures 1-3 also show that the reflective indices and $\varepsilon$ values of this system decreased linearly with the increase in temperature at the same mass content of the polymer derivative CS-AY GG, whereas $\beta$ increases linearly. The material is to be used as high-performance thermo-optic polymer devices with the consideration of the control of $n$ at appropriate temperature and the proper orientation of the constant.

In recent times, Fernández et $a l^{34}$ reported optically active multilayer films based on chitosan and an azopolymer, where the optical response of the developed polyelectrolyte multilayer (PEM) film-induced photo-orientation of photoactive azobenzene groups was giving rise to a birefringence in the film structure. The birefringence formation can be inferred by the change in transmittance of a probe beam that passes through the sample between crossed polarizers. From the above interesting results of chitosan-based optical materials, the researchers highlighted that chitosan-based materials can be used for applications in diverse biotechnological areas.

\section{Conclusion}

CS-AY GG complex showed optical properties with red shift. The SHG conversion efficiency of the derivative was observed greater than that of KDP. The $\mathrm{d} n / \mathrm{d} T$ and $\varepsilon$-values for the polymer were in the range $-2.5 \times 10^{-4}$ to $1.2 \times$ $10^{-4 \circ} \mathrm{C}^{-1}$ and 2.2 to 2.3 , respectively. The $\mathrm{d} n / \mathrm{d} T$ values were larger than that of inorganic glasses and $\varepsilon$-value was lower than that of the optically estimated values of conventional polymer. The obtained chitosan derivative was expected to be useful for optical switching and optical waveguide areas for biomedical device applications.

\section{References}

1. Cui Y, Ren H, Yu J, Wang Z and Qian G 2009 Dyes Pigm. 81 53

2. Qiu F, Xu H, Cao Y, Jiang Y, Zhou Y, Liu J and Zhang X 2007 Mater. Charact. $\mathbf{5 8} 275$

3. Bruno V, Castaldo A, Centore R, Sirigu A, Sarcinelli F, Casalboni M and Pizzoferrato R 2002 J. Polym. Sci. Part A: Polym. Chem. 401468 
4. Yang Y, He Q, Duan L, Cui Y and Li J 2007 Biomaterials 28 3083

5. Jiang H, Su W, Caracci S, Bunning T J, Cooper T and Adams W W 1996 J. Appl. Polym. Sci. 611163

6. Bhowmik P K, Kamatam S, Han H and Nedeltchev A K 2008 Polymer 491748

7. Teng C C 1992 Appl. Phys. Lett. 601538

8. Mortazavi M A, Knoesen A, Kowel S T, Henry R A, Hoover J M and Linsay G A 1991 Appl. Phys. B 53287

9. Singer K D, Kuzyk M G, Holland W R, Sohn J E, Lalama S L, Comizzoli R B, Katz H E and Schilling M L 1988 Appl. Phys. Lett. 531800

10. Girton D G, Kwiatkowski S L, Lipscomb G L and Lytel R S 1991 Appl. Phys. Lett. 581730

11. Yang J Y, Zhou Q J and Chen R T 2002 Appl. Phys. Lett. 81 2947

12. Hida Y, Onose H and Imamura S 1993 IEEE Photon. Technol. Lett. 5782

13. Eldada L, Stengel K M T, Shacklette L W, Norwood R A, Xu C Z, Wu J C and Yardley J T 1997 Proc. SPIE 3006344

14. Waldeck B 1993 Chirality 5350

15. Ghosh S, Banthia A K and Maiya B G 2002 Org. Lett. 43603

16. Ohya Y, Takei T, Kobayashi H and Ouchi T 1993 J. Microencapsulation 101

17. Angiolini L, Benelli T, Giorgini L, Mauriello F and Salatelli E 2007 Macromol. Chem. Phys. 2081548

18. Han S H 2004 J. Korean Phys. Soc. 451169

19. Demarger-Andre S and Domard A 1994 Carbohydr. Polym. 23 211
20. Singh J, Kumar S and Dutta P K 2009 J. Polym. Mater. 26 167

21. Kumar S, Kiran, Dutta P K and Koh J 2014 Int. J. Polym. Mater. Polym. Biomater. 63173

22. Archana D, Dutta J and Dutta P K 2014 Asian Chitin J. 101

23. Mehta A S, Singh B K, Singh N, Archana D, Snigdha K, Harniman R, Rahatekar S S, Tewari R P and Dutta P K 2015 J. Biomater. Appl. 291314

24. Archana D, Singh B K, Dutta J and Dutta P K 2015 Int. J. Biol. Macromol. 7349

25. Kumar S, Nigam N, Ghosh T, Dutta P K, Singh S P, Mishra L and Datta P K 2009 J. Polym. Mater. 26411

26. Clegg W (ed) 1998 The intensity of diffracted X-rays (Oxford Science Publication) p 21

27. Kumar S and Dutta P K 2008 Asian Chitin J. 467

28. Kumar S, Dutta J and Dutta P K 2009 J. Macromol. Sci. Pure Appl. Chem. A 461095

29. Dhanaraj P V, Rajesh N P and Bhagavannarayana G 2010 Physica B 4053441

30. Li W B, Segre P N, Gammon R W, Sengers J V and Lamvik M J 1994 J. Chem. Phys. 1015058

31. Watanabe Y, Shibasaki Y, Ando S and Ueda M 2005 Polymer 465903

32. Ando S, Watanabe Y and Matsuura T 2002 Jpn. J. Appl. Phys. 415254

33. Matsumoto T 1999 Macromolecules 324933

34. Fernández R, Ocando C, Fernandes S C M, Eceiza A and Tercjak A 2014 Biomacromolecules 151399 